\title{
MICROANGIOPATHIC COMPLICATIONS IN LATENT AUTOIMMUNE DIABETES IN ADULTS (LADA) - RELATIONSHIP WITH THYROID AUTOIMMUNITY
}

Authors: Aura Diana Reghina',2, Elisabeta Sava', Suzana Florea', Simona Fica'

1. Elias University Hospital, Endocrinology and Diabetes Department, UMF Carol Davila, Bucharest, Romania

2. National Institute of Development and Research in Pathology and Biomedical Sciences "Victor Babes",

Bucharest, Romania

Background:

Patients with TA had twice the risk of RD compared to patients without TA, odds ratios $2.27(95 \% \mathrm{Cl} 0.8$ la 5.7$), p=0.004$.

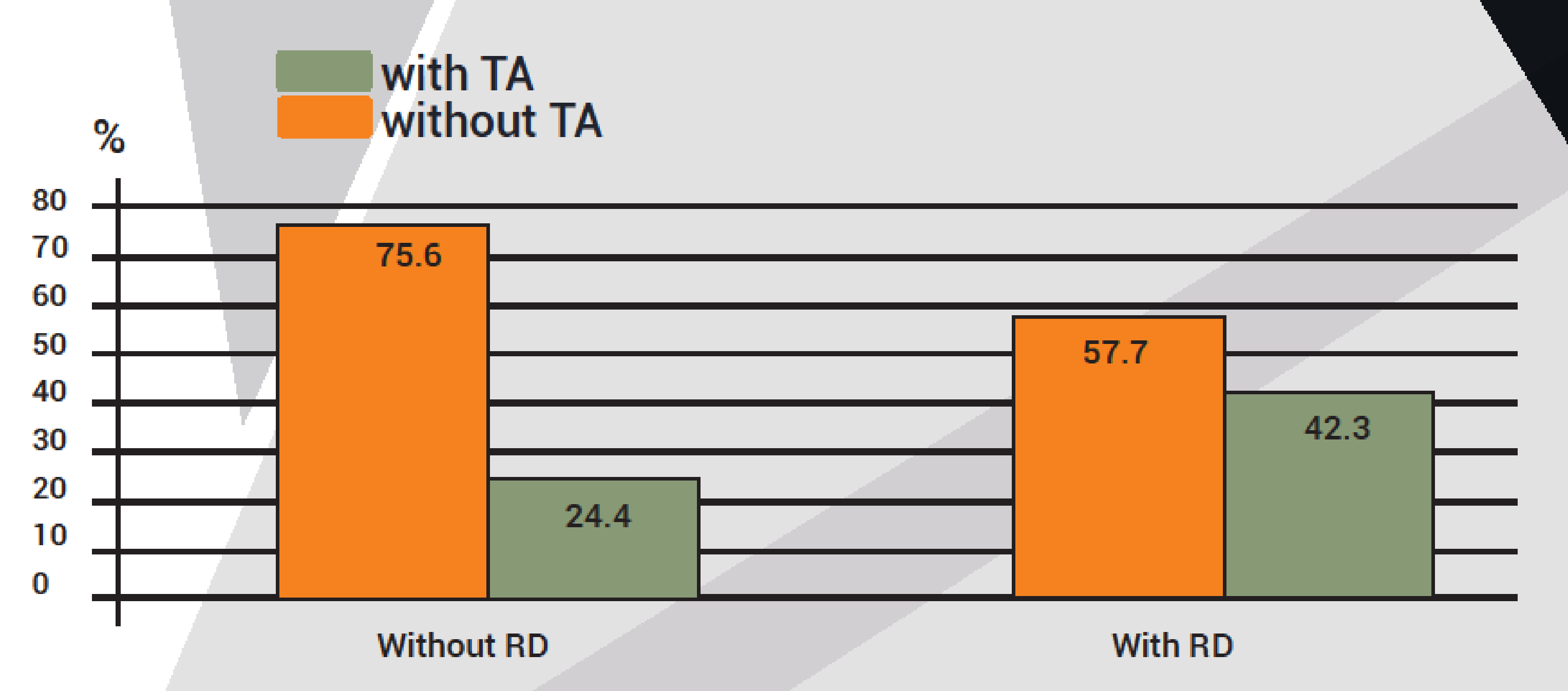

The risk of NP was 1.8 times higher in patients with TA compared with patients without TA $(95 \% \mathrm{Cl} 0.7$ la $4.4 ; \mathrm{p}=0.04)$.

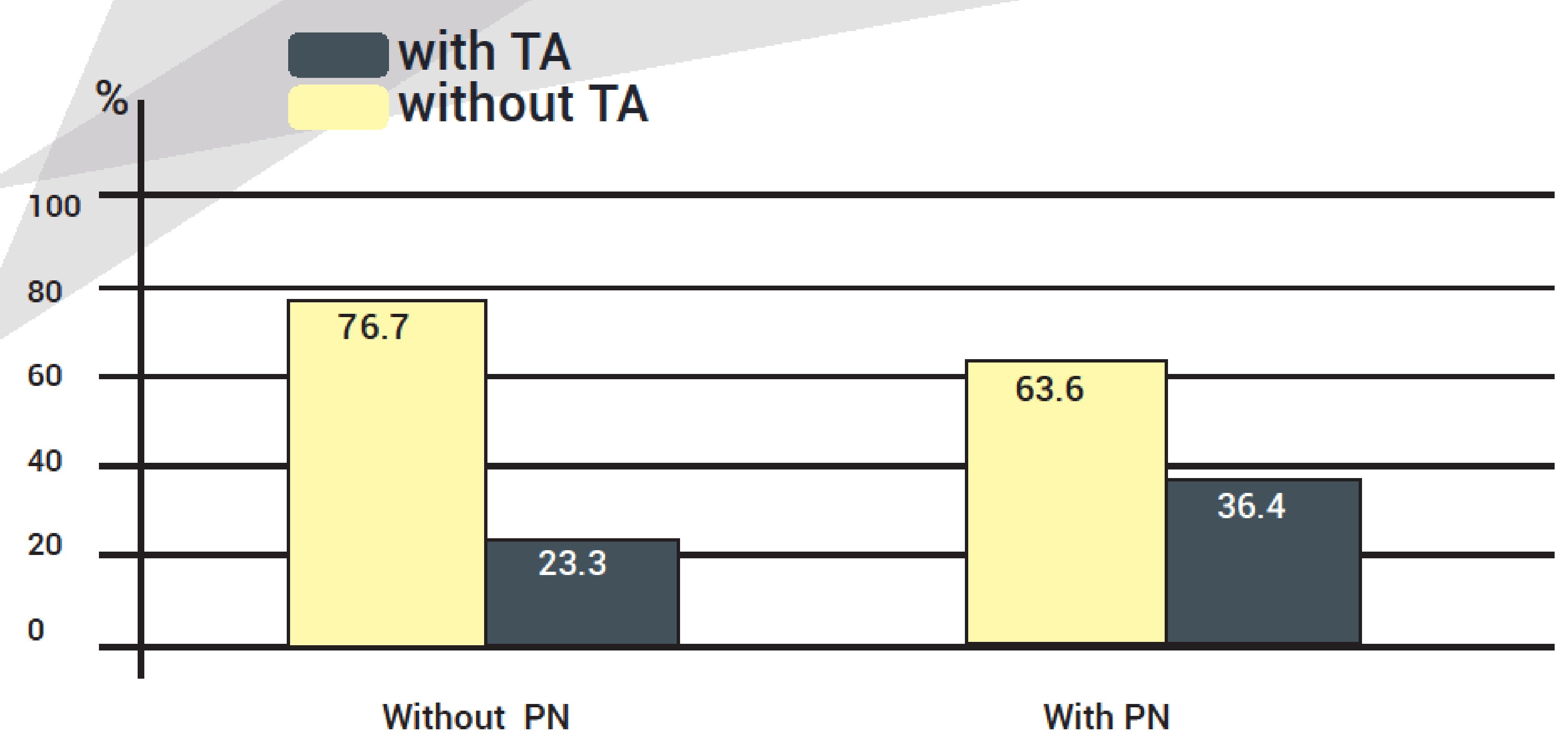

From 10 patients with DN, 4 of them (40\%) had TA compared to 26 patients $(27.7 \%)$ of 94 without DN. Patients with TA had the probability to develop this complication 1.7 times higher than those without $\mathrm{TA}$, odds ratio $1.74(95 \% \mathrm{Cl} 0.4$ la $6.6, \mathrm{p}=0.46)$.

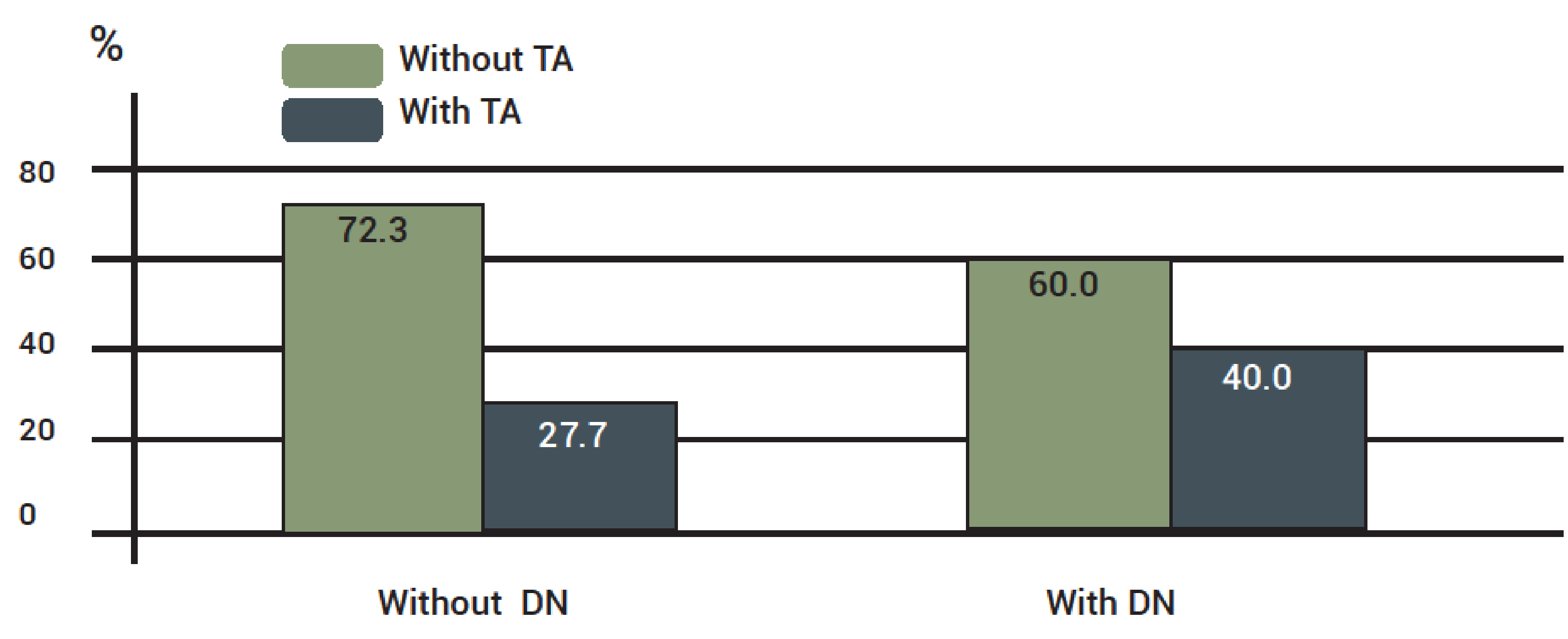

Patients with TA are three times more likely to develop AN than patients without TA, odds ratio $3.4(95 \% \mathrm{Cl} 1$ la 11, $\mathrm{p}=0.014)$.

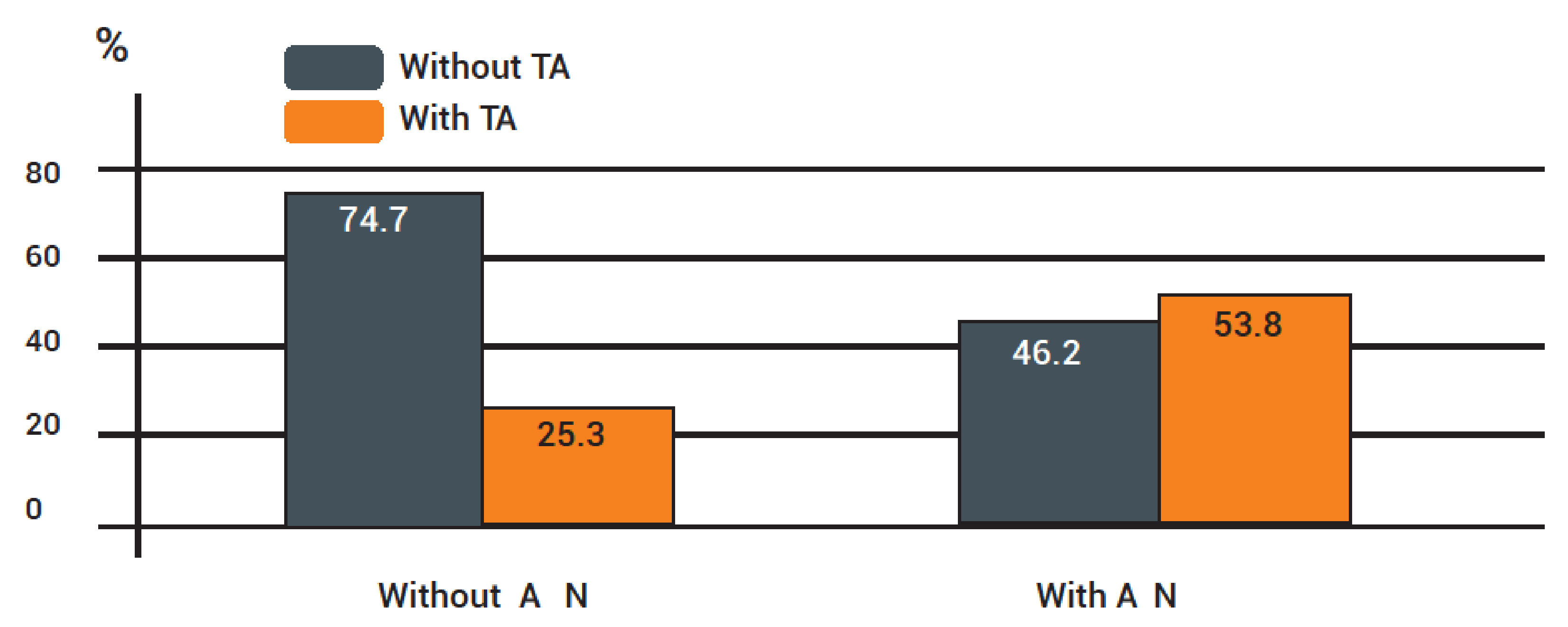

Conclusions:

Except diabetic nephropathy, the presence of thyroid autoimmunity in patients with LADA has determined a significantly higher prevalence of microangiopathic complications.

ACKNOWLEDGEMENT: This paper is partly supported by the Sectorial Operational Programme Human Resources Development (SOPHRD), financed by the European Social Fund and the Romanian Government under the contract number POSDRU 141531"

\section{Material and method:}

LADA has been defined by the onset of diabetes at ages above 30 years, the lack of need for insulin therapy for at least 6 months after its first appearance, the presence of anti GAD antibodies (GADA). Positive titers of TPOAb (anti-thyroid peroxidase antibodies ) were observed in $30(28.8 \%)$ out of 104 LADA patients. Prevalence of retinopathy (DR), peripheral neuropathy (PN), autonomic neuropathy (AN) and diabetic nephropathy (DN) were also evaluated. 